\title{
Corela
}

Cognition, représentation, langage

$16-2 \mid 2018$

Vol. $16, n^{\circ} 2$

\section{Complexité, éléments et groupes consonantiques en berbère tachelhit}

\section{Mohamed Lahrouchi}

\section{OpenEdition}

\section{Journals}

\section{Electronic version}

URL: http://journals.openedition.org/corela/7097

DOI: $10.4000 /$ corela.7097

ISSN: 1638-573X

\section{Publisher}

Cercle linguistique du Centre et de l'Ouest - CerLICO

\section{Electronic reference}

Mohamed Lahrouchi, «Complexité, éléments et groupes consonantiques en berbère tachelhit », Corela [Online], 16-2 | 2018, Online since 07 December 2018, connection on 01 May 2019. URL : http:// journals.openedition.org/corela/7097 ; DOI : 10.4000/corela.7097

This text was automatically generated on 1 May 2019.

\section{(c) (i) (3) (2)}

Corela - cognition, représentation, langage est mis à disposition selon les termes de la licence Creative Commons Attribution - Pas d'Utilisation Commerciale - Partage dans les Mêmes Conditions 4.0 International. 


\title{
Complexité, éléments et groupes consonantiques en berbère tachelhit
}

1

\author{
Mohamed Lahrouchi
}

\section{Introduction}

1 Hooper (1976: 204) oppose la hiérarchie de force à celle de sonorité (cf. Sievers 1881, Jespersen 1904, Saussure 1916 et Clements 1990, entre autres) : les segments les plus forts dans sa hiérarchie s'avèrent être les moins sonores. L'auteur dresse, ainsi, pour les consonnes une échelle en haut de laquelle figurent les occlusives sourdes, considérées comme plus fortes que les occlusives sonores et les fricatives sourdes, elles-mêmes plus fortes que les fricatives sonores. Viennent ensuite les nasales, puis les liquides. Et en bas de l'échelle apparaissent les glides.

2 Plusieurs théories phonologiques reprennent cette hiérarchie de force mais l'expriment en termes de complexité. Parmi elles figure la théorie des Éléments dont les bases ont été posées dans les travaux de Kaye, Lowenstamm \& Vergnaud (1985), Harris (1990, 1994), et Backley (2011) (voir aussi la phonologie de dépendance, Anderson \& Ewen 1987 et la phonologie des particules, Schane 1984). La complexité s'y mesure en nombre d'éléments qu'un segment contient : plus le segment contient d'éléments, plus il est complexe.

3 Faisant suite au travail de Lahrouchi $(2009,2010)$ sur la structure interne des racines trilitères en berbère tachelhit, cet article propose d'apporter un éclairage supplémentaire sur la nature des consonnes qui composent ces racines. Il sera notamment question du contenu phonologique des consonnes obstruantes et des consonnes sonantes; deux classes naturelles qui jouent un rôle majeur dans l'organisation segmentale des racines en tachelhit. L'objectif est de montrer que la fonction de tête de racine attribuée aux consonnes obstruantes se justifie par leur complexité phonologique.

4 L'article s'organise en quatre sections : en $\$ 1$, nous aborderons brièvement l'hypothèse de tête-complément dans les racines trilitères en tachelhit. En \$2, nous examinerons le 
contenu des consonnes obstruantes et des consonnes sonantes, à la lumière de la théorie des éléments. La notion de complexité apportera, à cet égard, un argument supplémentaire quant à la place des obstruantes dans la racine. En §3, nous évoquerons le rôle que peuvent avoir certains éléments consonantiques. Notre hypothèse sera que tous les éléments qui définissent les consonnes ne se valent pas, et que ceux disposés en haut d'une hiérarchie prédéterminée et universellement motivée renforcent la structure des consonnes auxquelles ils participent. Nous récapitulerons nos conclusions en $\S 4$.

\section{La structure interne des racines trilitères}

\subsection{Une structure hiérarchique binaire tête - complément}

Dans une étude récente (Lahrouchi 2010), réalisée sur 220 verbes comprenant des trilitères de type $\mathrm{CCC}, \mathrm{CCU}, \mathrm{CCI}$ et des bilitères de type $\mathrm{CC}$, exception faite des emprunts à l'arabe comme $f h m$ « comprendre », $x d m$ « travailler » et $H k m$ « juger », l'auteur note que $94 \%$ des verbes contiennent au moins une sonante, laquelle est précédée dans $84 \%$ des cas d'une obstruante. Les exemples en (1) illustrent les différents types de verbes retenus, classés en quatre groupes selon la nature obstruante ou sonante de leur consonnes ( 0 pour obstruante, $S$ pour sonante) :

(1)

\begin{tabular}{|l|l|}
\hline OOS & \\
\hline bsr & « étaler » \\
\hline zgr & «traverser » \\
\hline gzm & « couper » \\
\hline kSm & « entrer » \\
\hline Oso & \\
\hline krf & « attacher » \\
\hline krz & «labourer » \\
\hline Hrg & « brûler » \\
\hline frd & « démanger » \\
\hline & \\
\hline SOS & \\
\hline mgr & « moissonner » \\
\hline lkm & « arriver » \\
\hline
\end{tabular}




\begin{tabular}{|l|l|}
\hline$m^{\mathrm{I}} l$ & « enterrer » \\
\hline$n k r$ & « se lever» \\
\hline & \\
\hline oss & \\
\hline knu & « se pencher » \\
\hline bri & « égratigner » \\
\hline xmr & « fermenter » \\
\hline zlu & « perdre » \\
\hline
\end{tabular}

6 Rares en berbère sont les verbes comme bdg "mouiller » et bzg "enfler » qui ne contiennent que des obstruantes. Ceux qui sont entièrement constitués de sonantes comme $r m i$ «être fatigué » et $m l u$ "être mou» ne représentent pas plus de $9 \%$ des données examinées.

7 Partant de ces observations, l'auteur propose que les racines trilitères possèdent une structure hiérarchique binaire, dotée d'une tête et d'un complément. ${ }^{2}$ Dans cette structure, similaire à celles utilisées dans les représentations syntaxiques ou syllabiques, la consonne obstruante et la sonante qui la suit constituent, respectivement, la tête et le complément. Elles partagent le même nœud dans l'arbre. Le troisième segment qui compose la racine est un segment satellite, attaché à un nœud supérieur. Il peut apparaître aussi bien à gauche qu'à droite des consonnes tête et complément. De plus, il est libre d'être de type sonant ou obstruant.

Pour illustrer le modèle, quelques unes des racines en (1) sont représentées en (2) :

(2)

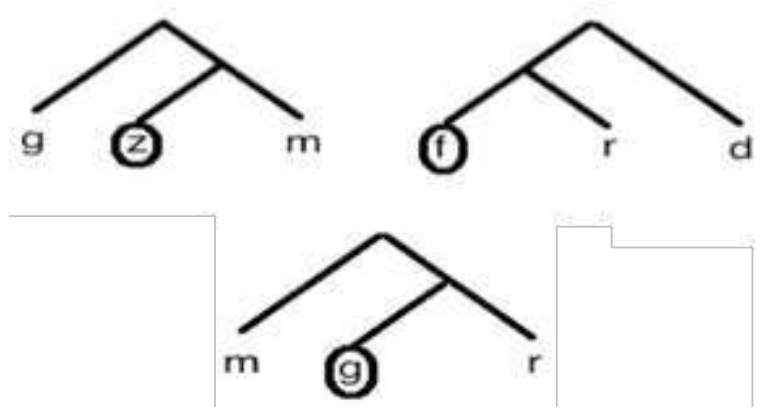

9 Comme il apparaît dans ces représentations, la fonction de tête de la racine est attribuée à l'obstruante (entourée) immédiatement à gauche de la sonante. Dans les racines dépourvues d'obstruantes comme rmi « être fatigué » et mlu " être mou », qui pour rappel représentent seulement $9 \%$ des racines examinées, ou dans celles de type knu «se pencher ", une sonante peut apparaître en position de tête. Dans ce cas, la tête est localisée immédiatement à gauche du segment le plus sonore, comme indiqué en (3) :

(3) 

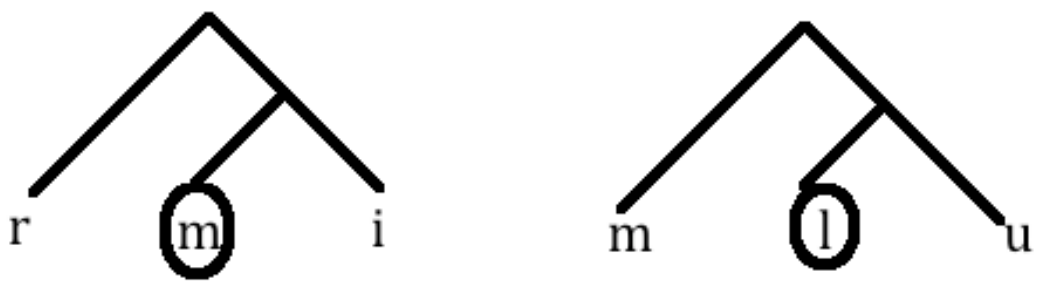

Dans la section suivante, nous montrons comment le modèle qui vient d'être brièvement exposé rend compte d'un des mécanismes morphologiques les plus productifs en berbère tachelhit, à savoir la gémination au thème de l'inaccompli.

\subsection{La gémination au thème de l'inaccompli}

11 La gémination est l'une des trois opérations que le berbère en général, et le tachelhit en particulier, utilisent pour former le thème de l'inaccompli. Elle concerne les verbes qui ne comptent pas plus de trois consonnes sans voyelles pleines et les verbes de type CCU et CCI. Plusieurs études, parmi lesquelles Bensoukas (2001), Boukous (1987), Cadi (1987), Chaker (1973, 1984), Chami (1979), Dell \& Elmedlaoui (1988, 1991, 2002), Hammane (2010), Jebbour (1996, 1999), Lahrouchi $(2001,2008,2010)$ et Louali \& Philipson (2004) se sont intéressées à ce phénomène, essentiellement en tachelhit. À la différence des autres variétés du berbère où la gémination à l'inaccompli touche invariablement la consonne médiane, en tachelhit c'est tantôt la consonne médiane qui gémine, tantôt l'initiale.

Nous reprenons ci-dessous les exemples en (1), accompagnés de leurs formes à l'inaccompli :

(4)

\begin{tabular}{|l|l|l|}
\hline Racine & Inaccompli & \\
\hline oOS & & \\
\hline bsr & bssr & « étaler » \\
\hline zgr & zggr & « traverser » \\
\hline gzm & gzzm & « couper » \\
\hline kSm & kJSm & « entrer » \\
\hline & & \\
\hline OSO & & \\
\hline krf & kkrf & « attacher » \\
\hline krz & kkrz & « labourer » \\
\hline Hrg & HHrg & « brûler » \\
\hline
\end{tabular}




\begin{tabular}{|c|c|c|}
\hline frd & ffrd & « démanger» \\
\hline SOS & & \\
\hline mgr & mggr & «moissonner» \\
\hline $1 \mathrm{~km}$ & $1 \mathrm{kkm}$ & « arriver» \\
\hline $\mathrm{md}^{\mathrm{S}} 1$ & $m t t^{\mathrm{S}} 1$ & « enterrer» \\
\hline $\mathrm{nkr}$ & nkkr & "se lever" \\
\hline oss & & \\
\hline knu & knnu & «se pencher» \\
\hline bri & brri & « égratigner » \\
\hline $\mathrm{xmr}$ & $\mathrm{xmmr}$ & «fermenter » \\
\hline 3lu & 3llu & «perdre» \\
\hline
\end{tabular}

13 Seuls les verbes de type OSO géminent la consonne initiale à l'inaccompli ; les autres géminent la consonne médiane. Dans le modèle exposé ici, cette variation s'obtient naturellement comme le résultat d'une opération qui cible la tête de la racine et que l'on peut formuler comme en (5):

(5) Pour dériver l'inaccompli, il faut géminer la consonne qui est en position de tête de la racine.

14 A ce stade, cette analyse ne fait guère mieux que les analyses syllabiques classiques (Dell \& Elmedlaoui 1988, 1991, 2002, 2013). Mais l'intérêt premier de l'hypothèse tête complément réside dans le fait qu'elle prédit que tous les verbes et seulement les verbes qui ont une structure binaire tête - complément forment l'inaccompli par gémination. Autrement dit, elle explique non seulement la gémination dans les verbes comme en (4), mais aussi l'absence de gémination dans les verbes comme bdg "être mouillé » et $r k z$ « danser » que Dell \& Elmedlaoui (2002: 118) appellent d'ailleurs 'geminable verbs'. Alors que l'hypothèse syllabique prédit incorrectement que ces verbes géminent la consonne médiane, notre analyse dit qu'ils n'ont tout simplement pas de consonne tête identifiée qui puisse géminer à l'inaccompli. ${ }^{3}$ Il est intéressant de noter qu'en tachelhit les verbes de ce type forment leur inaccompli par préfixation de $\mathrm{tt}-.^{4}$

La structure hiérarchique binaire tête-complément, érigée autour de l'obstruante et de la sonante, semble donc non seulement déterminer les racines trilitères sujettes à la gémination à l'inaccompli, mais distingue aussi les racines qui géminent la consonne initiale de celles qui géminent la consonne médiane (cf. Dell \& Elmedlaoui 2013 pour une discussion critique de ce modèle). Une question importante reste néanmoins posée: qu'estce qui justifie dans la structure hiérarchique adoptée le choix des obstruantes comme consonnestêtes au détriment des sonantes? 
Les arguments distributionnels et morphologiques évoqués jusqu'ici appuient certes la structure hiérarchique, mais risquent d'être interprétés comme des arguments circulaires, en ce sens qu'on ne connaît pas le lien de cause à effet entre la fonction-tête des obstruantes et le fait qu'elles géminent à l'inaccompli. Il nous faut pour asseoir l'hypothèse tête-complément dans les racines trilitères trouver d'autres arguments qui justifient le rôle structurant des obstruantes et des sonantes, mais surtout le choix des obstruantes comme consonnes-têtes. La section suivante en fournira quelques uns.

\section{Obstruante - Sonante}

\subsection{Quelques arguments dans et en dehors du domaine berbère}

17 Les premiers arguments en faveur du modèle proposé pour les racines trilitères viennent des racines bilitères et monolitères. En effet, ayant défendu que les trilitères possèdent une structure hiérarchique binaire, dans laquelle l'obstruante et la sonante constituent respectivement la tête et le complément de la racine, nous nous attendons à ce que les racines bilitères soient composées seulement d'une obstruante suivie d'une sonante. Autrement dit, d'une tête, suivie de son complément.

18 Sur les 26 racines bilitères examinées (voir la liste dans Lahrouchi 2010), 13 ont effectivement la forme OS (ex. gn « dormir », $f l$ « laisser », $d^{i} r$ « tomber ») et 6 sont de type SO (ex. ls « s'habiller», $n s$ " passer la nuit », $r z^{\varepsilon}$ " casser »); les autres sont entièrement composées de sonantes (ex. $m l$ «montrer», $n u$ « être cuit») ou d'obstruantes (ex. $s B$ " acheter », $b z$ « creuser »). Notons, cependant, que la plupart des racines qui dérogent à la structure OS se comportent comme de vraies trilitères. Les exemples ci-dessous illustrent ce point :

(6)

\begin{tabular}{|l|l|l|l|l|}
\hline & Aoriste & Inaccompli & Accompli (3ms) & \\
\hline a. & ls & lssa & i-lsa & «s'habiller » \\
\hline & ns & nssa & i-nsa & «passer la nuit » \\
\hline nz & nzza & i-nza & « se vendre » \\
\hline b. & bnu & bnnu & i-bna & « construire » \\
\hline & 3lu & 3llu & i-3la & «perdre » \\
\hline & dru & drru & i-dra & «partager le repas » \\
\hline
\end{tabular}

19 Les verbes bilitères en (6a) présentent plusieurs similitudes avec les trilitères en (6b) :

- À l'inaccompli, ils géminent tous la seconde consonne radicale.

- À l'accompli comme à l'inaccompli, ils se terminent par la voyelle -a.

Ceci a poussé plusieurs auteurs à considérer ces verbes comme fondamentalement trilitères (voir entre autres Dell \& Elmedlaoui 1988, Iazzi 1991, 2018, Lahrouchi 2001). 
uant aux racines monolitères, on s'attend à ce qu'elles soient formées seulement d'obstruantes, c'est-à-dire de la consonne-tête. Les racines de ce type que nous avons trouvées sont effectivement toutes formées d'obstruantes : $g$ " être », kk « passer par », $\int$ «manger », $f$ « donner ». L'obstruante est en quelque sorte à la racine ce qu'une voyelle est à la syllabe ou encore ce qu'un verbe est au syntagme verbal (VP) : de la même façon qu'une syllabe peut n'être constituée dans l'écrasante majorité des langues que d'une voyelle et qu'un VP peut n'être composé que d'un verbe, une racine peut ne contenir en berbère qu'une consonne obstruante.

En dehors du domaine berbère, le bella coola, langue amérindienne de Colombie britannique connue pour tolérer des groupes consonantiques complexes, présente un cas intéressant. Bagemihl (1991) rapporte que dans cette langue, la suite obstruante-sonante joue un rôle essentiel dans l'organisation syllabique des segments. Toute suite de segments qui ne répond pas au patron syllabique obstruante-sonante-voyelle-consonne reste non-syllabée. De plus, la réduplication qui sert à exprimer le diminutif ou le continuatif, consiste généralement à répéter dans le mot exactement la suite obstruantesonantes : par exemple, tqnk > tqnqnk «être sous", st'qwlus > st'qwlqwlus "piège pour l'ours noir » (Bagemihl 1991 : 203).

\subsection{La complexité segmentale}

Depuis quelques décennies, sont apparues des théories phonologiques qui définissent, partiellement ou totalement, le contenu des segments en termes de traits ou primitives unaires, dits aussi monovalents. La théorie des éléments en fait partie (voir entre autres Kaye, Lowenstamm \& Vergnaud [KLV] 1985, 1988, Harris 1990, 1994, Scheer 1996, Backley 2011). ${ }^{5}$ Elle rejette pour le trait non seulement son caractère binaire, mais aussi sa fonction phonologique (voir aussi la phonologie de dépendance (Anderson \& Ewen 1987), et la phonologie des particules (Schane 1984). Pour reprendre les termes de KLV (1988: 110) :

Le constituant ultime de cette théorie n'est pas le trait phonologique (...) L'unité première qui constitue le segment est l'élément, lequel est une matrice de traits entièrement spécifiée, phonétiquement interprétable.

À ses débuts, la théorie des éléments a été appliquée essentiellement aux systèmes vocaliques (anglais : Harris 1994, finnois, français : Kaye 2000, kpokolo : KLV 1988, Kabyle : Bendjaballah 1995). Trois éléments vocaliques sont distingués : |I, A, U|, auxquels s'ajoute une voyelle froide (neutre), notée $\left|\mathrm{v}^{\circ}\right|$ dans certaines versions de la théorie (KLV 1985, Scheer 1996, Harris 1990) et |@| dans d'autres (Harris 1994). Ces éléments, entièrement spécifiés, prononçables phonétiquement et caractérisables acoustiquement ${ }^{6}$, peuvent apparaitre seuls ou se combiner dans des expressions complexes, être en de position tête ou en position d'opérateur, afin de dériver différentes voyelles. Pour prendre quelques exemples concrets: l'élément $|\mathrm{I}|$ seul, ou associé à la voyelle froide |@|, dérive phonétiquement [i]. De même, $|\mathrm{U}|$ associé à $|@|$ dérive $[\mathrm{u}]$. Et $|\mathrm{A}|$ associé à $|@|$ dérive $[\mathrm{a}]$. En revanche, lorsque $|\mathrm{A}|$ s'associe à $|\mathrm{I}|$, le résultat peut être [e] ou [æ] suivant que $|\mathrm{A}|$ ou $|\mathrm{I}|$ est la tête de l'expression. D'autres combinaisons possibles, empruntées à Harris (1994), apparaissent ci-après (l'élément-tête est soulignée):

(7)

$$
\begin{array}{|l|l|}
\hline|\underline{\mathrm{U}}, \mathrm{A}|=[\mathrm{o}] & |\underline{@}, \mathrm{I}|=[\mathrm{I}] \\
\hline
\end{array}
$$




\begin{tabular}{|l|l|}
\hline$|\underline{\mathrm{A}}, \mathrm{U}|=[\mathrm{p}]$ & $|\underline{@}, \mathrm{U}|=[\mathrm{\sigma}]$ \\
\hline$|\underline{@}, \mathrm{~A}|=[\mathrm{e}]$ & $|\underline{@}|=[\partial]$ \\
\hline
\end{tabular}

Ces mêmes éléments vocaliques, associés à d'autres éléments, ont été intégrés dans la composition des consonnes. Leur nombre et leur nature diffèrent légèrement d'un auteur à l'autre, mais l'essentiel reste le même (voir en particulier Harris 1994, Scheer 1996, Kaye 2000, Backley 2011). Pour les besoins de l'analyse, nous utiliserons les éléments en (8), que nous empruntons à Harris (1994) :

(8)

\begin{tabular}{|l|}
\hline$|\mathrm{A}|:$ position centrale de la langue \\
\hline$|\mathrm{I}|:$ palatalité \\
\hline$|\mathrm{U}|:$ labialité \\
\hline$|@|:$ vélarité \\
\hline$|\mathrm{P}|:$ occlusion \\
\hline$|\mathrm{h}|:$ bruit, friction \\
\hline$|\mathrm{R}|:$ coronalité \\
\hline$|\mathrm{N}|:$ nasalité \\
\hline$|\mathrm{H}|:$ cordes vocales tendues (consonnes non-voisées) \\
\hline$|\mathrm{L}|:$ cordes vocales relâchées (consonnes voisées) \\
\hline
\end{tabular}

Chaque élément renvoie à un lieu ou à un mode d'articulation. |A, I, U, @| définissent le lieu d'articulation ${ }^{7}$. Le lecteur se référera au travail original (Harris 1994) pour une justification phonétique et phonologique de ces éléments. Nous nous intéresserons, pour notre part, à la différence qui peut exister en termes d'éléments entre les obstruantes et les sonantes. Ce qui nous ramène à la question que nous avons soulevée précédemment, et que nous rappelons ci-dessous :

- Qu'est-ce qui justifie dans la structure hiérarchique adoptée pour les racines trilitères le choix des obstruantes comme consonnes-têtes?

Le principe de complexité, proposé par Harris (1990: 274) et KLV (1990:218), arrive à point nommé. Établi initialement pour rendre compte de certaines relations de gouvernement, ce principe stipule que les segments les plus complexes gouvernent les segments les moins complexes (pour une présentation des principes de la phonologie de gouvernement, voir KLV 1990 et Scheer 2004). La complexité d'un segment se définit en termes du nombre d'éléments qu'il contient: plus le segment contient d'élément, plus il est complexe. Par exemple, dans les langues ayant des attaques branchantes de type $f l, p l$ et $g r$, le principe de complexité permet aux consonnes obstruantes de gouverner les sonantes. ${ }^{8}$ 
Appliqué au cas qui nous intéresse ici, le principe de complexité explique pourquoi les racines trilitères en berbère tachelhit privilégient les obstruantes en position de tête plutôt que les sonantes : les obstruantes tirent leur légitimité à occuper la position de tête de la racine du fait qu'elles sont plus complexes que les sonantes. Autrement dit, elles contiennent plus d'éléments que les sonantes. Pour illustrer cette idée, comparons en (9) le contenu des obstruantes $/ \mathrm{kg} \int \mathrm{fb} /$ à celui des sonantes $/ \mathrm{wj} / \mathrm{r} \mathrm{n} /$.

(9)

\begin{tabular}{|l|l|l|}
\hline Obstruantes & Sonantes \\
\hline $\mathrm{k}|\mathrm{P} @ \mathrm{~h} \mathrm{H}|$ & $\mathrm{r}|\mathrm{R}|$ \\
\hline $\mathrm{g}|\mathrm{P} \mathrm{@} \mathrm{h} \mathrm{L}|$ & $\mathrm{I} \mid \mathrm{R}$ ? \\
\hline $\mathrm{S}|\mathrm{h} \mathrm{R} \mathrm{I} \mathrm{H}|$ & $\mathrm{n}|\mathrm{N}|$ \\
\hline $\mathrm{f}|\mathrm{h} \mathrm{U} \mathrm{H}|$ & $\mathrm{w}|\mathrm{U}|$ \\
\hline $\mathrm{b} \mid \mathrm{h} \mathrm{U}$ ? L $\mid$ & $\mathrm{j}|\mathrm{I}|$ \\
\hline
\end{tabular}

Harris (1994), auquel nous empruntons ces structures, justifie le contenu des consonnes en grande partie par des processus de lénition. Nous invitons le lecteur à se référer à son travail pour le détail des arguments. Signalons, à titre d'exemple, la lénition en coréen de $p$ en $w$ et de $t$ en $r$ à l'intervocalique :

(10)

\begin{tabular}{|l|l|l|}
\hline Indicatif & statif & \\
\hline ki:p-t'a & kiw-ə & « être froid» \\
\hline tə:p-t'a & təw-ə & « être chaud» \\
\hline mu:t-t'a & mur-ə & « questionner » \\
\hline kə:t-t'a & kər-ə & « marcher » \\
\hline
\end{tabular}

Partant de l'idée que la lénition est un processus d'affaiblissement qui entraîne une perte du contenu segmental, les exemples en (10) suggèrent que $p$ et $t$ sont plus complexes que $w$ et $r$. En termes d'éléments, cette lénition se traduit par la perte des éléments d'occlusion $|\mathrm{P}|$ et de bruit $|\mathrm{h}|$ dans $p$ et $t$, laissant seulement l'élément labial $|\mathrm{U}|$ qui se réalise en $w$ et l'élément coronal $|\mathrm{R}|$ qui se réalise en $r$. Des cas similaires existent en anglais australien : got to > ['gDrə] « devoir » (Backley 2011 : 130).

Un autre cas intéressant nous vient du rifain. Dans cette variété berbère parlée au nord du Maroc, les liquides subissent une lénition qui transforme /l/ en [r] et qui efface /r/ (cf. Chtatou 1982, Dell \& Tangi 1993, Amrous 1999, Amrous \& Bensoukas 2003, Iazzi 2018). Les exemples ci-dessous illustrent ce phénomène :

(11) 


\begin{tabular}{|c|c|c|c|}
\hline & Forme phonologique & Forme phonétique & \\
\hline a. & /argaz/ & a:gaz & « homme» \\
\hline & /ad'ar/ & да: & « jambe » \\
\hline & /atbir/ & 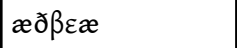 & "pigeon» \\
\hline & $/ z^{\mathrm{q}} u \mathbf{r}^{\mathrm{q}} /$ & $z^{\mathrm{q}} \mathrm{oa}$ & « visiter» \\
\hline & /nad'or/ & naðoa & « Nador» \\
\hline b. & $/ \mathrm{ul} /$ & ur & « cœur » \\
\hline & /ulinu/ & urinu & «mon cœur » \\
\hline & /xlf/ & xrəf & « remplacer» \\
\hline & /ils/ & irəs & « langue» \\
\hline & /bla/ & bra & «sans» \\
\hline
\end{tabular}

En (11a), /r/ s'efface en position de coda et provoque au passage un allongement compensatoire de la voyelle précédente (ex. a:gaz) ou une dightongaison (ex. $\left.\mathrm{z}^{\mathrm{i}} \mathrm{oa}\right)$. En (11b), /l/ se réalise toujours [r], qu'il soit en position de coda (ex. ul > ur « cœur») ou d'attaque (ex. ulinu > urinu «mon cœur »). ${ }^{9}$ Cette trajectoire de lénition, appelé traditionnellement "rhotacisme ", se lit parfaitement dans les structures proposées en (9) : un / $\mathrm{r} /$ n'est rien d'autre qu'un / $/$ privé de son élément d'occlusion $|\mathrm{P}|$. Ceci est schématisé en (12) ci-après. Fait plus intéressant encore, les deux consonnes contiennent peu d'éléments comparés aux consonnes obstruantes

(12)

\begin{tabular}{|l|l|l|l|l|}
\hline \multicolumn{5}{|c|}{ Rhotacisme en berbère rifain } \\
\hline$/ \mathrm{l} /$ & $=$ & $/ \mathrm{r} /$ & $=$ & $\varnothing$ \\
\hline$|\mathrm{R}|$ & & $|\mathrm{R}|$ & & $|\mathrm{R}|$ \\
\hline$|\mathrm{P}|$ & & $|\mathrm{H}|$ & & \\
\hline
\end{tabular}

Le fait que les obstruantes contiennent plus d'éléments que les sonantes justifie, de notre point de vue, leur apparition en position de tête de la racine. Toute racine consonantique qui contient une suite OS se voit donc attribuer une structure hiérarchique binaire où l'obstruante est la tête et la sonante qui suit son complément. Rappelons, tout de même, qu'une sonante peut être tête en l'absence d'une obstruante, et seulement dans le cas des racines contenant au moins deux sonantes successives comme dans $m r z$ «blesser à la tête », lmd « apprendre », rmi « être fatigué » et nru « vaincre ». 
Notre analyse pourrait s'arrêter ici. Mais avant, il convient de dire un mot sur les limites du principe de complexité dans l'analyse du contenu mélodique de certaines classes de consonnes.

\section{La géométrie des éléments}

Si le principe de complexité suffit à expliquer que les obstruantes sont globalement plus complexes que les sonantes, il reste insuffisant pour établir une hiérarchie entre les consonnes qui appartiennent à une même classe. Dans la classe des obstruantes, par exemple, les consonnes $/ k g \int t d b /$ contiennent toutes le même nombre d'éléments, d'après Harris (1994) :

(13)

\begin{tabular}{|c|c|}
\hline $\mathrm{k}|\mathrm{l} @ \mathrm{hH}|$ & 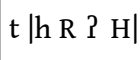 \\
\hline $\mathrm{g}|\mathrm{P} @ \mathrm{~h} \mathrm{~L}|$ & $\mathrm{d}|\mathrm{h} R \mathrm{R} \quad \mathrm{L}|$ \\
\hline $\int \mid \mathrm{h} \mathrm{R} \mathrm{I} \mathrm{H}$ & $\mathrm{b} \mid \mathrm{h}$ U ? L \\
\hline
\end{tabular}

Pourtant, elles ne se comportent pas de la même façon vis-à-vis des processus phonologiques. / $t /$ est, par exemple, connue pour être la consonne épenthétique par excellence : en français, elle apparaît dans des dérivations comme sirop > siroter, café > cafetière, ainsi que dans les inversions interrogatives. En cela, elle se distingue des autres consonnes obstruantes. Mais, la notion de complexité ne permet pas de capturer cette différence. Une alternative possible, au vu des expressions en (13), serait de postuler que la hiérarchie entre obstruantes tient compte non seulement du nombre d'éléments, mais aussi de la présence ou non d'un élément-tête dans l'expression : les obstruantes pourvues d'un élément-tête seraient alors considérées comme hiérarchiquement supérieures aux obstruantes dépourvues de tête.

Le raisonnement pourrait être poussé plus loin, au point de considérer qu'une hiérarchie existerait entre les éléments eux-mêmes. L'idée n'est pourtant pas nouvelle; elle soustend l'organisation géométrique des éléments proposée par Harris (1994:129) et Harris \& Lindsey (1995: 76), que nous leur empruntons ci-après en (14) :

(14) 


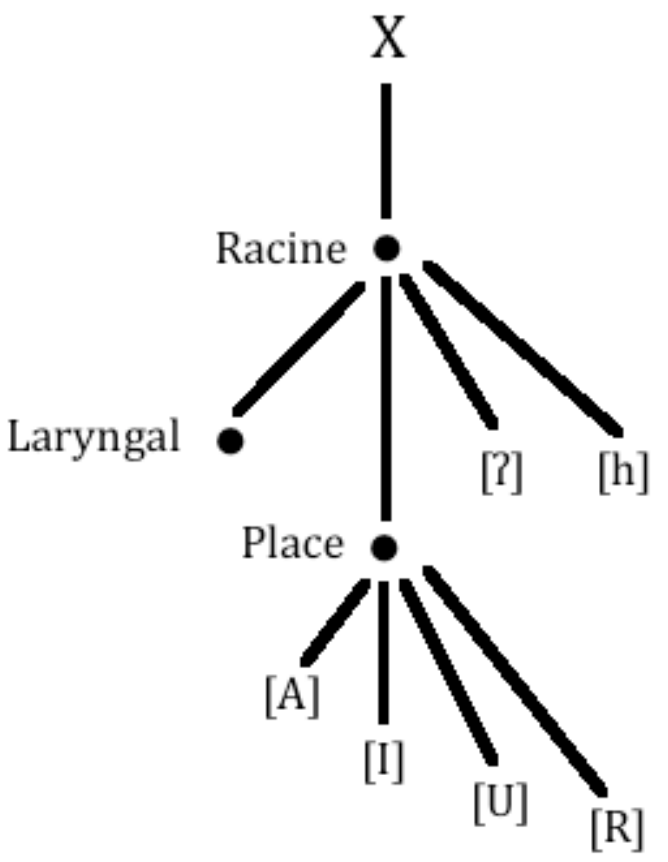

Comme les traits dans les arbres géométriques classiques (Clements 1985, Sagey 1986), les éléments sont dominés par des nœuds, notamment Racine, Place et Laryngal ${ }^{10}$. Une telle organisation géométrique est supposée rendre compte des processus phonologiques qui ciblent des éléments ou des nœuds spécifiques dans l'arbre. Par exemple, la débuccalisation qui transforme certaines occlusives en [?] (ex. anglais de Londres got > [go ?]) et certaines fricatives en [h] (ex. espagnol d'Amérique Centrale mes > meh «bouche »), consisterait simplement à supprimer dans l'arbre le noud de Place pour ne laisser que $|\mathrm{l}|$ et $|\mathrm{h}|$ sous Racine (voir aussi le malais où $/ \mathrm{p}, \mathrm{t}, \mathrm{k} />[\mathrm{?}]$ et $/ \mathrm{s}, \mathrm{f}, \mathrm{h} />[\mathrm{h}]$, Clements \& Hume 1995).

Une autre lecture possible de cette organisation géométrique consisterait à voir $|\mathrm{l}|$ et $|\mathrm{h}|$ comme des éléments hiérarchiquement supérieurs aux éléments de Place $|\mathrm{A} I \mathrm{U} R|$, notamment $d u$ fait qu'ils figurent plus haut dans l'arbre (14). Le processus de débuccalisation, que nous venons d'évoquer, semble appuyer cette lecture: en termes d'éléments, la débuccalisation revient à vider les consonnes de leur substance, en supprimant tous les éléments qu'elles contiennent, à l'exception de $|\mathrm{l}|$ et $|\mathrm{h}|$. Cela suggérerait donc que les obstruantes qui ont $|\mathrm{P}|$ ou $|\mathrm{h}|$ dans leur structure, de préférence en position de tête, sont plus fortes que les autres obstruantes. En réexaminant les expressions en (13), on constate que seul $/ \mathrm{J} /$ possède un de ces deux éléments en position de tête, tandis que $k$ et $g$ ont |@| comme tête et les autres consonnes sont dépourvues de tête. Il convient cependant de préciser que le contenu de certains segments diffère d'un auteur à l'autre. Ainsi, à l'inverse de Harris (1994), Backley (2011 : 138) considère qu'en anglais, $/ k /$ ainsi que toutes les autres obstruantes sourdes ont $|\mathrm{H}|{ }^{11}$ comme élément-tête tandis que / $g$ / est dépourvu de tête. Backley inclut, en plus, $|\mathrm{U}|$ dans le contenu de / $k /$ et / g/ (voir aussi Scheer 1996). Les arguments en faveur de cela ne manquent pas. En berbère, par exemple, les consonnes vélaires, mais aussi les uvulaires, sont sensibles au phénomène de labio-vélarisation comme le montrent les exemples suivants en tachelhit :

(15) 


\begin{tabular}{|l|l|l|l|}
\hline & Aoriste & Accompli & \\
\hline a. & knu & ikwna & "se pencher » \\
\hline & gru & igwra & « ramasser » \\
\hline & xlu & ixwla & " détruire » \\
\hline b. & dru & idra & "partager la nourriture » \\
\hline & bnu & ibna & « construire » \\
\hline & nru & inra & " vaincre » \\
\hline
\end{tabular}

41 A l'accompli, les verbes en (15a) conservent une trace de la voyelle $u$ qui se manifeste par la labialisation des consonnes vélaires et uvulaires. Les verbes en (15b) perdent toute trace de la voyelle labiale. ${ }^{12}$ Un autre argument en faveur de la présence de l'élément $|U|$ dans les vélaires nous vient $\mathrm{du}$ hausa. ${ }^{13}$ Dans cette langue tchadique, la voyelle interne - $a$ - (pénultième) du pluriel (16a) change en $u$ au voisinage d'une consonne vélaire (16b) :

(16)

\begin{tabular}{|l|l|l|l|}
\hline & Singulier & Pluriel & \\
\hline a. & kásá: & kásà:shé: & «terre » \\
\hline & táushì: & táfà:shé: & « type de tambour » \\
\hline & fárí: & fárá:ré: & « blanc » \\
\hline b. & kàrée & karnúkàa & « chien » \\
\hline & rá:mìi & rámúkà: & « fosse » \\
\hline & rà:fi: & rá:fúkà: & « ruisseau » \\
\hline
\end{tabular}

Ces exemples, apparentés aux pluriels internes bien connus dans les langues afroasiatiques, ne représentent qu'une classe de pluriels en haoussa. On compte dans cette langue pas moins de 46 classes de pluriels que Newman $(2000: 431)$ ramène à 15 classes principales (Voir aussi Hellwig \& Mcintyre 2000). Ce qui intéressant à propos des exemples en (16) c'est l'interaction qui existe entre la voyelle infixée /a/ au pluriel et les segments voisins. Cette interaction implique non seulement l'alternance de la voyelle infixée avec [u] lorsque la consonne voisine est une vélaire, mais aussi l'alternance de la voyelle finale /e/ avec [a] en réaction à la nature de la voyelle précédente. Á cela s'ajoute un changement des tons associés aux voyelles ; un phénomène que certains auteurs analysent comme une forme de corrélation entre tons et voyelles (cf. PilszczikowaChodak 1972, 1975, Lahrouchi 2004). 

structure des consonnes obstruantes. Les exemples de lénition que nous avons mentionnés montrent que ce sont les derniers éléments qui restent dans la structure des obstruantes lorsqu'elles s'affaiblissent en anglais $(t>$ ?), en espagnol $(s>h)$, mais aussi en malais ( $\mathrm{p}, \mathrm{t}, \mathrm{k}>\mathrm{?} / \mathrm{s}, \mathrm{f}, \mathrm{h}>\mathrm{h}$ ). En d'autres termes, $|\mathrm{P}|$ et $|\mathrm{h}|$ sont l'ultime stade de la lénition des obstruantes, avant qu'elles ne s'effacent complètement; d'où leur position haute dans l'arbre géométrique en (14). Les consonnes qui les possèdent en position de tête sont donc sensées être plus fortes qui les autres consonnes.

\section{Conclusion}

Dans ce travail, nous avons apporté quelques arguments supplémentaires en faveur de l'hypothèse tête-complément dans les racines trilitères en berbère tachelhit, initialement proposée par Lahrouchi $(2009,2010)$. Après un bref rappel de cette hypothèse, qui stipule que les racines trilitères contenant une suite obstruante-sonante possèdent une structure hiérarchique binaire où l'obstruante est la tête de la racine et la sonante qui suit, son complément, nous avons présenté des arguments qui justifient le rôle de tête des obstruantes. Dans le cadre de la théorie des éléments, nous avons vu que les obstruantes sont plus complexes que les sonantes; une complexité mesurée en termes du nombre d'éléments que les consonnes contiennent. Nous avons, ensuite, montré que la complexité à elle seule ne suffit pas à hiérarchiser toutes les consonnes, et que pour distinguer les obstruantes entre elles il fallait prendre en considération la présence d'un élément-tête dans la consonne, et surtout sa nature. En nous appuyant sur un modèle géométrique des

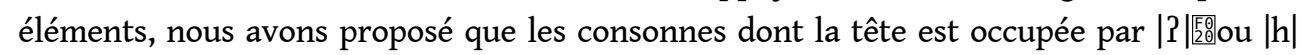
sont hiérarchiquement supérieurs (plus forts) que les autres consonnes.

\section{BIBLIOGRAPHY}

AMrous, Nourddine. 1999. Phonological Processes in Ait Oulichek Tarifiyt Berber. Unpublished Memoire paper, Mohammed 5 University, Rabat.

AMROUS, Nourddine \& Karim BENSOUKAS. 2003. Tarifit long vowels and diphtongs: Independent phonemes or simple phonetic variants of the basic Amazigh vowels?. In Standardisation de l'amazighe, Abdallah Boumalk \& Meftaha Ameur (eds), 117-139. Rabat: IRCAM Publications. ANDERSON, John. 1985. Structural analogy and dependency phonology. Acta Linguistica Hafniensia 19: 5-44.

ANDERSON, John. 2002. A notational theory of syntactic categories. Cambridge Studies in Linguistics 82. Cambridge: Cambridge University Press.

ANDERSON, John \& Colin EWEN. 1987. Principles of dependency phonology. Cambridge: Cambridge University Press.

Corela, $16-2$ | 2018 
ARCHANGELI, Diana. 1988. Underspecification in Yawelmani Phonology and Morphology. Outstanding Dissertations in Linguistics, Garland Publishing, New York.

BAGEMIHL, Bruce. 1991. Syllable structure in Bella Coola. Linguistic Inquiry 22: 589-646.

BACKLEY, Phillip. 2011. An introduction to Element Theory. Edinburgh: Edinburgh University Press. BENSOUKAS, Karim. 2001. Stem forms in the nontemplatic morphology of Berber. Doctoral dissertation, Université Mohammed 5, Rabat.

BENSOUKAS, Karim. 2014. Featural dissimilation in Tashlhit: Avoiding the repetition of labial and round. Rabat: University Mohamed 5.

Boukous, Ahmed. 1987. Phonotactique et domaines prosodiques en berbère. Thèse de Doctorat, Université Paris 8.

CADI, Kadour. 1987. Système verbal rifain, forme et sens. Paris: Selaf.

CHAKER, Salem. 1973. Le système dérivationnel verbal berbère (dialecte Kabyle). Thèse de Doctorat, Université Paris 3.

CHAKER, Salem. 1984. Système des oppositions verbales (kabyle), formes et valeurs. Textes en linguistique berbère: introduction au domaine berbère, 160-176. Paris: CNRS Editions.

CHAMI, Mohamed. 1979. Un parler amazighe du Rif marocain. Thèse de Doctorat, Université Paris 5. CHTATOU, Mohamed. 1982. Aspects of the Phonology of a Berber dialect of the Rif. PhD dissertation, London, University of London/SOAS.

CLEMENTS, G. Noam. 1985. The geometry of phonological features. Phonology Yearbook 2: 223-250. CLEMENTS, G. NOAM \& Elizabeth HUME. 1995. The internal organization of speech sounds. In The Handbook of Phonology, John Goldsmith (ed.), 245-306. Oxford : Blackwell.

DELL, François \& Mohamed ELMEdLAOUI. 1988. Syllabic consonants in Berber: some new evidence. Journal of African Languages and Linguistics 10: 1-17.

DELL, François \& Mohamed ELMEdLAOUI. 1991. Clitic ordering, morphology and phonology in the verbal complex of Imdlawn Tashlhiyt Berber. Langues Orientales Anciennes Philologie et Linguistique 3: $77-104$

DELL, François \& Mohamed ELMEDLAOUI. 2002. Syllables in Tashlhiyt Berber and in Moroccan Arabic. Dordrecht: Kluwer Academic Publishers.

DELL, François \& Oufae TANGI. 1993. On the vocalization of /r/ in Ath-Sidhar Rifian Berber.

Linguistica Communicatio 5/1-2: 5-53.

EL HAMDI, Fatima. 2018. On Tashlhit root structure and its implications for the organization of the lexicon. PhD dissertation, Mohammed 5 University \& Paris 8 University.

EL KIRAT, Yamina. 1987. Spirantization in the Beni Iznassen dialect, diachrony and synchrony. D.E.S.S.

Thesis, Mohammed 5 University, Rabat.

ELMEDLAOUI, Mohamed. 1992. Aspects des representations phonologiques dans certaines langues chamitosémitiques. Thèse de Doctorat d'Etat, Université Mohammed 5, Rabat.

HAMMANE-ABOUFARAH, Kadija. 2010. La syllable en berbère tachelhit: que peut apporter la théorie CVCV? Thèse de Doctorat, Université Paris 8.

HAMMOND, Michael. 1984. Constraining metrical theory: A modular theory of rhythm and destressing. Doctoral dissertation, UCLA, Los Angeles, CA. 
HARRIS, John. 1990. Segmental complexity and phonological government. Phonology Yearbook 7/2: 255-300.

HARRIS, John. 1994. English sound structure. Oxford: Blackwell.

HARRIS, John \& Geoff LINDSEY. 1995. The elements of phonological representations. In Frontiers of Phonology: Atoms, Structures, Derivations, Jacques Durand \& Francis Katamba (eds.), 34-79. London: Longman.

HELLWIG, Birgit \& A. Joseph MCINTYRE. 2000. Hausa plural Systems: A diachronic presentation. Journal of African Languages and Linguistics 21: 1-44.

HOOPER, Joan B. 1976. An introduction to Natural Generative Phonology. New York: Academic Press.

IAZzI, El Mehdi. 1991. Morphologie du verbe en tamazight (parler des Aït Attab, Haut Atlas Central): approche prosodique. Mémoire de D.E.S, Université Mohammed 5, Rabat.

IAZZI, El Mehdi. 2018. Norme et variations en amazighe marocain (aspects morpho-phonologiques). Thèse de Doctorat d'Etat, Université Ibn Zohr, Agadir.

JEBBOUR, Abdelkrim. 1996. Morphologie et contraintes prosodiques en berbère (tachelhit de Tiznit): analyse linguistique et traitement automatique. Thèse de Doctorat, Université Mohammed 5, Rabat. JEBBOUR, Abdelkrim. 1999. Syllable weight and syllable nuclei in Tashlhiyt Berber of Tiznit. Cahiers de Grammaire 24 : 95-116.

JESPERSEN, Otto. 1904, Lehrbuch der Phonetik. Leipzig and Berlin: B. G. Teubner.

KAYE, Jonathan. 2000. A Users' Guide to Government Phonology. Ms., University of Ulster.

KAYE, Jonathan, Jean LOWENSTAMM \& Jean-Roger VERGNAUD. 1985. The internal structure of phonological elements: a theory of charm and government. Phonology Yearbook 2: 305-328.

KAYE, Jonathan, Jean LOWENSTAMM \& Jean-Roger VERGNAUD. 1988. La structure interne des elements: une théorie du Charme et du Gouvernement. Recherches Linguistiques de Vincennes 17 : 109-134.

KAYE, Jonathan, Jean LOWENSTAMM \& Jean-Roger VERGNAUD. 1990. Constituent structure and government in phonology. Phonology Yearbook 7: 193-231.

LAHROUCHI, Mohamed. 2001. Aspects morpho-phonologiques de la dérivation verbale en berbère (parler chleuh d'Agadir). Thèse de Doctorat, Université Paris 7.

LAHROUCHI, Mohamed. 2004. La corrélation tons-voyelles dans certains pluriels en haoussa. Communication aux $6^{\text {èmes }}$ Journées du Réseau Français de Phonologie, 3-4 juin, Grenoble.

LAHROUCHI, Mohamed. 2008. A templatic approach to gemination in the imperfective stem of Tashlhiyt Berber. Studies in African Linguistics 37/1: 21-60.

LAHROUCHI, Mohamed. 2009. La structure interne des racines triconsonantiques en berbère tachelhit. In Etudes de phonétique et de linguistique berbères. Hommage à Naïma Louali 1961-2005, Salem Chaker, Amina Mettouchi \& Gérard Philippson (eds), 177-193, M-S. Ussun amazigh 23 / SELAF, Editions Peeters, Paris / Louvain.

LAHROUCHI, Mohamed 2010. On the internal structure of Tashlhiyt Berber triconsonantal roots. Linguistic Inquiry 41/2: 255-285.

LOUALI, Naïma \& Gérard PHILIPPSON. 2004. Le thème de l'aoriste intensif: formes multiples, contenu unique. In Nouvelles Etudes Berbères, Le Verbe et autres Articles, Kamal Naït-ZERRAD, Rainer Vossen \& Dymitr IBRISZIMOW (eds), 79-94. Frankfort: Rüdiger Köppe Verlag. 
NEWMAN, Paul. 2000. The Hausa Language: An Encyclopedic Reference Grammar. New Haven, London: Yale University Press.

PILSZCZIKowA-CHODAK, Nina. 1972. Tone-Vowel Height Correlation and Tone Assignment in the Patterns of Verb and Noun Plurals in Hausa. Studies in African Linguistics 3/3: 399-421.

PILSZCZIKOWA-CHODAK, Nina. 1975. On the Correlation of Tone and Vowel Height in Hausa: a Replay to Newman. Studies in African Linguistics 6/3: 315-321.

PRINCE, Alan. 1985. Improving tree theory. In Proceedings of the Eleventh Annual Meeting of the Berkeley Linguistics Society, Mary Niepokuj, Mary VanClay, Vassiliki Nikiforidou \& Deborah Feder (eds), 471-490. Berkeley: University of California, Berkeley Linguistics Society.

SAGEY, Elizabeth. 1986. The representation of features and relations in nonlinear phonology. $\mathrm{PhD}$ dissertation, MIT.

SAUSSURE, Ferdinand de. 1916. Cours de linguistique générale. Paris, Payot.

Schane, Stanford. 1984. The fundamentals of Particle Phonology. Phonology Yearbook 1: 129- 155.

SCHEER, Tobias. 1996. Une théorie de l'interaction directe entre consonnes. Thèse de Doctorat, Université Paris 7.

SCHEER, Tobias. 2004. A Lateral Theory of Phonology: What is CVCV, and why should it be?. Studies in Generative Grammar 68.1. Berlin: Mouton de Gruyter.

SELKIRK, Elisabeth. 1993. [Labial] relations. Manuscript, University of Massachusetts, Amhrest.

SIEVERS, Eduard. 1881. Grundzüge der Phonetik. Leipzig: Breitkopf \& Hartel.

SOUAG, Lameen. 2010. Grammatical contact in the Sahara. PhD dissertation, SOAS, London.

TILMATINE, Mohamed. 1996. Un parler berbèro-songhay du sud-ouest algérien (Tabelbala) :

Eléments d'histoire et de linguistique. Etudes et Documents Berbères 14 : 163-198.

\section{NOTES}

1. Je remercie pour leurs commentaires et propositions les évaluateurs anonymes et les éditeurs de la revue. Il est bien évident que je demeure seul responsable des erreurs que pourrait contenir cet article.

2. Sur les notions de "tête » et de " complément » et leur usage en phonologie, voir entre autres Anderson $(1985,2002)$ et Anderson \& Ewen (1987) dans le cadre de phonologie de dépendance, Kaye, Lowenstamm \& Vergnaud $(1985,1990)$ dans le cadre de la phonologie du Gouvernement et Hammond (1984) et Prince (1985) dans le cadre de la phonologie métrique et accentuelle.

3. Un évaluateur pose judicieusement la question de la structure des racines qui contiennent une voyelle en position initiale ou médiane. En l'état actuel, l'hypothèse tête-complément ne s'applique pas à la structure des racines de ce type. Elle prédit seulement qu'elles ne formeraient pas leur inaccompli par gémination vu qu'elles ne possèdent pas la structure hiérarchique adéquate. En cela, l'hypothèse tête-complément ne fait pas mieux que l'hypothèse syllabique classique selon laquelle les verbes de type mun «être ramassé » et arud « être lavé » devraient logiquement géminer la consonne en position d'attaque pour former leur inaccompli. Dell \& Elmedlaoui (1988 : 11) affirment dans ce sens que "not all geminable verbs resort to gemination in the imperfective but most of them do". Ils ajoutent que "the distribution of the geminating verbs among the geminable verbs seems to be a matter of lexical idiosyncrasy." La raison pour laquelle les verbes à voyelle initiale ou médiane se comportent différemment des verbes à voyelle 
finale réside vraisemblablement dans la nature même des voyelles qu'ils contiennent. Á l'inverse des verbes de type knu «se pencher » et rmi «être fatigué » pour lesquels la voyelle finale peut être analysée comme un glide sous-jacent, situé sur le même pallier que les autres consonnes de la racine, la voyelle des verbes comme mun et arud est une voyelle stable (non-alternante) qui fait partie intégrante de la racine. Le lecteur est invité à lire le travail d'El Hamdi (2018) à propos de ce type de racines qu'elle nomme «racines vocaliques» opposées aux «racines consonantiques ».

4. Á ceux-là s'ajoutent les verbes empruntés à l'arabe comme xdm «travailler» et fhm " comprendre ». Ils forment en tachelhit un système à part du fait qu'ils résistent à la gémination à l'inaccompli alors même qu'ils possèdent la structure binaire tête-complément. Dans les autres variétés du berbère, comme le tarifit et le tamazight, ces verbes s'alignent sur les verbes trilitères natifs en formant leur inaccompli par gémination.

5. Voir aussi les représentations géométriques des traits (Clements 1985, Clements \& Hume 1995, Sagey 1986), où seuls les nœuds terminaux conservent des traits binaires, et la théorie de la sousspécification (Archangeli 1988) qui restreint le nombre de traits binaires.

6. Voir Harris (1994) sur les propriétés acoustiques des éléments vocaliques.

7. |I, A, U, @| ne sont pas les seuls éléments communs aux consonnes et aux voyelles. L'élément | $\mathrm{N} \mid$ utilisé dans les consonnes nasales, se retrouve aussi dans les voyelles nasales. De même, $|\mathrm{H}|$ et $\mid$ L qui caractérisent les consonnes voisées et non-voisées, servent aussi à marquer les tons hauts et bas.

8. Ces attaques branchantes, habituellement appelées attaques à sonorité ascendantes, sont largement attestées dans les langues naturelles, comme par exemple en français flocon, plateau, grue ou en anglais fly « voler », plane « avion », grow " grandir ».

9. Pour des faits similaires en kwarandzyey (Tabelbala, sud-ouest Algérie), voir Tilmatine (1996) et Souag (2010).

10. Dans un souci de simplifier le raisonnement, nous avons omis d'inclure dans l'arbre l'élément nasal $|\mathrm{N}|$ et les éléments $|\mathrm{H}|$ et $|\mathrm{L}|$ qui marquent, entre autres, les consonnes voisées et nonvoisées. L'élément nasal figurerait, d'après Harris (1994:129), sous le nœud Racine, juste à côté des éléments $|\mathrm{P}|$ et $|\mathrm{h}|$, tandis que les deux autres éléments apparaitraient sous le nœud Laryngal. 11. $|\mathrm{H}|$ est un élément de bruit et de friction dans le modèle de Backley (2011). Il équivaut ainsi à l'élément $|\mathrm{h}|$ chez Harris (1994).

12. Pour une analyse différente du phénomène de labio-vélarisation, le lecteur peut se référer aux travaux de Elmedlaoui (1992), Selkirk (1993) et Bensoukas (2014).

13. D'autres arguments qui montrent que les consonnes vélaires et uvulaires contiennent l'élément |U| sont fournis dans le travail de Scheer (1996).

\section{ABSTRACTS}

This article provides new evidence for the head-complement hypothesis in Tashlhiyt verbal roots. Originally proposed to account for the distribution of consonants in triliteral roots and their behaviour towards gemination in the imperfective stem (Lahrouchi 2009, 2010), the headcomplement hypothesis holds that roots containing an obstruant-sonorant sequence display a binary branching structure, where the obstruant is the head and the sonorant its complement. The complexity condition, which refers to the number of elements each segment contains, is used here to explain why the obstruents qualify for the head position in the root. In cases where 
complexity does not work, element geometry is mobilized to show that consonants headed by $\mid$ ? $\mid$ or $|\mathrm{h}|$ are stronger than other consonants.

Cet article apporte des arguments supplémentaires en faveur de l'hypothèse tête-complément dans les racines verbales en tachelhit. Initialement proposée pour rendre compte de la distribution des consonnes dans les racines trilitères ainsi que de la gémination au thème de l'inaccompli (Lahrouchi 2009, 2010), cette hypothèse stipule que les racines contenant une suite obstruante-sonante possèdent une structure hiérarchique binaire où l'obstruante est la tête de la racine et la sonante son complément. Pour justifier le rôle de tête des consonnes obstruantes, cet article évoque le principe de complexité. Dans le cadre de la théorie des éléments, les consonnes obstruantes sont considérées comme plus complexes que les sonantes; la complexité étant mesurée en termes du nombre d'éléments qui constituent le segment : plus le segment contient d'éléments, plus il est complexe. Dans les cas où la complexité s'avère insuffisante, il est proposé que les consonnes dont la tête est occupée par l'élément $\mid$ ? $\mid$ ou $|\mathrm{h}|$ sont plus fortes que les autres

\section{INDEX}

Mots-clés: phonologie, éléments, consonnes, racines, berbère.

Keywords: phonemics, elements, consonants, roots, Berber language

\section{AUTHOR}

\section{MOHAMED LAHROUCHI}

CNRS - Université Paris 8 